Revue des patrimoines

La Première Guerre mondiale vue de Paris : de la correspondance d'Étienne de Nalèche, directeur du Journal des Débats, à Pierre Lebaudy, au « journal d'un bourgeois de Paris », 1914-1919

\title{
Odile Gaultier-Voituriez
}

\section{(2) OpenEdition}

\section{Journals}

Electronic version

URL: http://journals.openedition.org/insitu/11727

DOI: 10.4000/insitu. 11727

ISSN: 1630-7305

\section{Publisher}

Ministère de la Culture

\section{Electronic reference}

Odile Gaultier-Voituriez, « La Première Guerre mondiale vue de Paris : de la correspondance d'Étienne de Nalèche, directeur du Journal des Débats, à Pierre Lebaudy, au « journal d'un bourgeois de Paris », 1914-1919», In Situ [Online], 25 | 2014, Online since 30 January 2015, connection on 25 June 2020 URL : http://journals.openedition.org/insitu/11727 ; DOI : https://doi.org/10.4000/insitu. 11727

This text was automatically generated on 25 June 2020 .

In Situ Revues des patrimoines est mis à disposition selon les termes de la licence Creative Commons Attribution - Pas d'Utilisation Commerciale - Pas de Modification 4.0 International. 


\title{
La Première Guerre mondiale vue de Paris : de la correspondance d'Étienne de Nalèche, directeur du Journal des Débats, à Pierre Lebaudy, au « journal d'un bourgeois de Paris ", 1914-1919
}

\author{
Odile Gaultier-Voituriez
}

1 La Première Guerre mondiale a été l'occasion d'une production écrite très abondante qui constitue de nombreuses sources pour en écrire l'histoire, d'un point de vue administratif ou événementiel, sur le terrain ou à l'arrière, immédiatement ou après la guerre. Il s'agit notamment des livres, des journaux ou des archives administratives. Une autre source a été redécouverte et exhumée relativement récemment: ce sont les écrits du for privé. Correspondances et carnets de guerre donnent à voir et à comprendre les sentiments personnels, la perception immédiate des événements grâce aux témoignages individuels écrits. Souvent très vivants et émouvants, peu volumineux par rapport à la richesse de leur contenu, ils ont été conservés dans les familles et parfois redécouverts récemment par la troisième ou la quatrième génération. Certains ont été remis à des institutions publiques pour numérisation lors de la Grande Collecte. Ces lettres, cartes, notes ou carnets ont été rédigés par des scripteurs d'un niveau d'instruction déjà assez élevé car ils sont capables de coucher sur le papier leur vie quotidienne et parfois leurs sentiments. Une immense part de ces sources est produite par les poilus ou leur famille, par le biais de leur correspondance mutuelle et des carnets de combattants. Les circonstances de la production varient également : le soldat et l'officier sont au front ou dans un état-major, leur famille est à l'arrière. Le niveau de langue diffère d'un auteur à l'autre ; le contenu et l'intérêt aussi. La conservation des documents - intacts dans leurs enveloppes ou couvertures d'origine ou classés, leur transmission aux descendants, leur remise à un service d'archives ou une bibliothèque, leur publication éventuelle changent évidemment le niveau de leur connaissance, leur valorisation et leur publicisation. 
2 La source évoquée maintenant constitue un cas particulier dans ce large panorama. Il s'agit bien d'écrits du for privé, une correspondance rédigée de 1914 à 1919 par Étienne de Nalèche, directeur du Journal des Débats, resté à Paris, qui s'adresse à son ami Pierre Lebaudy, industriel sucrier et officier qui s'est engagé malgré son âge. Une édition critique de ces lettres a été établie ${ }^{1}$. Le contexte et les circonstances de la production seront présentés dans un premier temps. Puis seront évoqués le corpus, sa conservation et sa transmission jusqu'à nos jours. Enfin, nous insisterons sur l'originalité du statut et la spécificité typologique de la source, entre épistolaire et journal, et sur ses apports : la guerre vue de Paris.

\section{Le contexte et les circonstances de la production}

Les deux correspondants sont des notables parisiens. Comte romain, Étienne de Nalèche est rompu aux usages du monde. Pendant cinquante ans, il dirige le très sérieux Journal des Débats fondé par les frères Bertin en 1789 (fig. 1). Il possède un réseau très étendu dans le Tout-Paris. Très riche industriel sucrier, Pierre Lebaudy est issu d'une famille originale comptant un empereur auto-proclamé du Sahara. Il possède un yacht, chasse en Afrique avant guerre, finance la construction de dirigeables pour l'armée au début du siècle, soutient beaucoup d'œuvres caritatives. Il fait construire pour son usage personnel un bel hôtel particulier rue François- ${ }^{\text {er }}$. Son épouse fait édifier après sa mort mais selon ses plans l'église du Sacré-Cœur des étudiants de la Cité universitaire à Gentilly, il est enfin actionnaire et financeur du Journal des Débats.

Figure 1

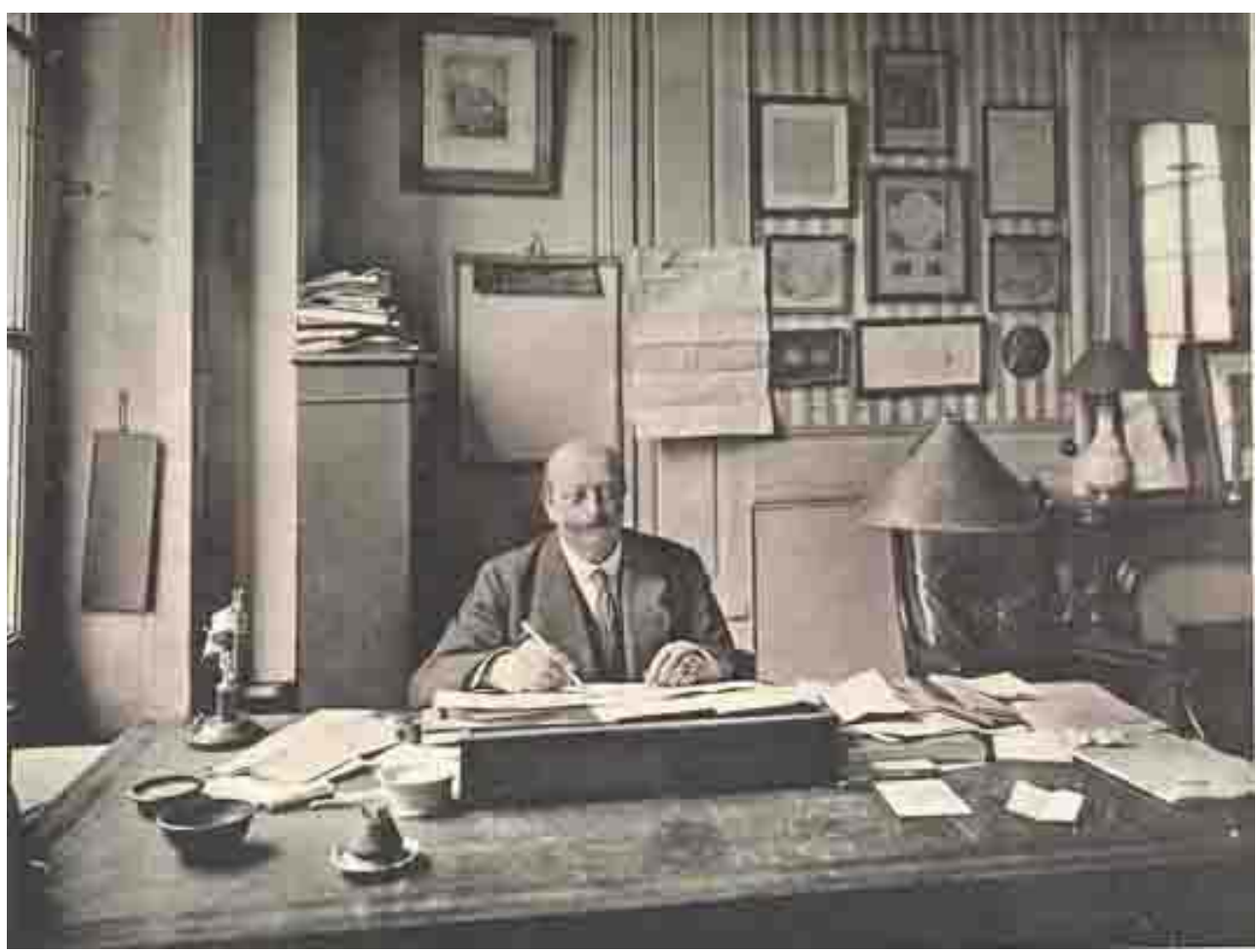

«Étienne de Nalèche dans la salle de rédaction du Journal des Débats, vers 1914 ». Alfred Pereire. Le Journal des Débats politiques et littéraires, 1814-1914. Paris : E. Champion, 1924, p. 160. Tous droits réservés. 
Figure 2

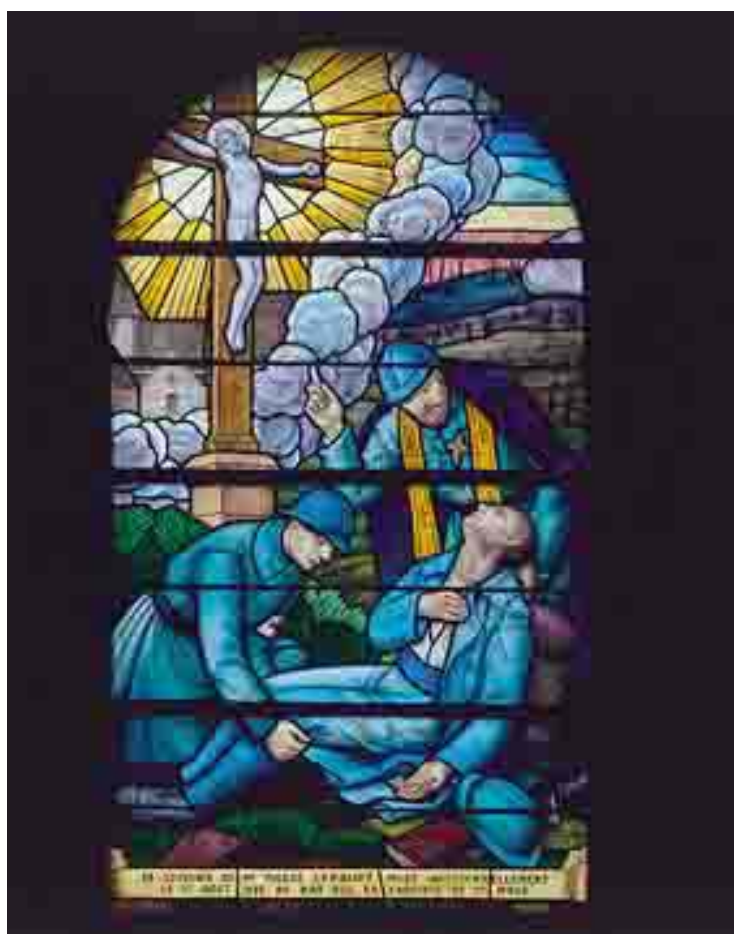

«En souvenir de M. Pierre Lebaudy mort accidentellement le $1^{\text {er }}$ août 1929 au Mas sur la paroisse de Saint-Maur ». Verrière historiée par Roger Desjardin, peintre-verrier, Angers, 1930, dans l'église de Saint-Maur (Cher). Un aumônier et un brancardier militaires de la guerre de 1914-1918 assistent aux derniers instants de leur compagnon ; apparition de la Croix dans une nuée ; au fond une église et un paysage dévasté par la guerre.

Phot. Malnoury, Robert, Martin, J. - (c) Inventaire général, ADAGP (IM 8000700 ; IVR24_95180262VA)

(fig. 2) ${ }^{2}$

Nalèche et Lebaudy ont de nombreux points communs. Ils sont issus de milieux proches, même si Nalèche est originaire d'une famille de robe anoblie et Lebaudy un riche industriel et grand bourgeois. Ils ont tous deux des racines provinciales (Creuse et Normandie), sont Versaillais dans leur enfance, puis Parisiens à l'âge adulte. Leur père à chacun était député, de la même couleur politique et à la même époque. Ils sont camarades d'enfance, de la même génération: nés en 1865, ils ont la cinquantaine au début de la guerre, ils se tutoient et s'appellent par leur prénom. La formule de politesse très affectueuse de la première lettre du corpus atteste de la proximité du lien ${ }^{3}$. Ils sont mariés et sans enfants. Ils partagent un grand nombre de valeurs, d'idées et de préoccupations, comme le goût pour l'art classique et les beaux objets. Ils sont intéressés par les questions politiques et économiques d'intérêt général, même si Nalèche est peutêtre plus attiré, de par son passé d'ancien diplomate, par les relations internationales, et Lebaudy, de par ses fonctions, est hostile à l'étatisme. Tous deux sont républicains modérés de centre droit et favorables au libéralisme. Leurs intérêts économiques convergent. Nalèche est probablement associé aux affaires sucrières de son ami. Pierre Lebaudy est actionnaire de la Compagnie universelle du canal de Suez dont Nalèche est administrateur à partir de 1918. C'est très certainement Nalèche qui a suggéré à son beaufrère Pierre de Vauréal de «faire appel à quelques amis fidèles dénommés "groupe étranger ${ }^{4}$ » ", dont Lebaudy, pour le faire entrer en 1907 dans le capital de la Compagnie des phares ottomans, co-fondée par le grand-père de l'épouse de Nalèche. Cette 
convergence d'intérêts économiques au Moyen-Orient explique aussi leur intérêt commun pour les questions diplomatiques dans cette partie du monde. Beaucoup plus riche que Nalèche, Lebaudy est associé aux différentes entreprises de celui-ci, en grande partie pour les financer. L'analyse du profil de nos deux correspondants et du lien tissé entre eux - amitié d'enfance, partage de valeurs et conjonction d'intérêts économiques nous aide à percevoir ce qu'est réellement leur correspondance pendant la guerre de 1914. Les deux hommes font alors des choix différents: tous deux ont dépassé l'âge obligatoire pour combattre, mais Lebaudy, officier de réserve, s'engage et est capitaine à l'état-major d'une division territoriale d'infanterie ${ }^{5}$, tandis que Nalèche fait le choix de rester à Paris. Ce dernier commence sa correspondance pour se rapprocher de Lebaudy dont il est séparé. Les lettres sont destinées à abolir la distance, malgré le décalage entre ce qu'ils vivent.

\section{Le corpus, sa conservation et sa transmission jusqu'à nos jours}

6 Jean-Noël Jeanneney, professeur des universités à l'Institut d'études politiques de Paris, avait eu l'occasion de travailler sur les lettres de Nalèche à Lebaudy pour sa thèse et son ouvrage consacrés à l'industriel et homme politique François de Wendel, un des financeurs du Journal des Débats ${ }^{6}$. Il en avait mesuré tout l'intérêt et la richesse. À notre connaissance, le fonds a été relativement peu utilisé tant que la famille le détenait. Outre Jean-Noël Jeanneney, Pierre Miquel pour son article et son livre sur le traité de Versailles ${ }^{7}$ et ses notes des souvenirs de Poincaré et Gilbert Joseph pour sa biographie de Fernand de Brinon $^{8}$ 'en sont servi ponctuellement.

7 De manière inhabituelle, le corpus était conservé par la famille Nalèche - et non par celle du destinataire, Lebaudy, ce qui laisse supposer que Nalèche avait conscience de l'intérêt de son fonds ou que Mme Lebaudy, après la mort brutale de son mari en 1929 dans un accident de voiture, avait renvoyé ses missives au scripteur. Cela signifie de toutes manières une volonté de conservation et c'est une pratique fréquente depuis le XVIII siècle. Après négociations, les documents prêtés par Mme Henri de Nalèche, nièce par alliance d'Étienne de Nalèche, à Pierre Miquel et Gilbert Joseph ont pu être rassemblés. Mme Henri de Nalèche a fait don des lettres à la Fondation nationale des Sciences politiques (FNSP) ${ }^{9}$ le 14 mai 1997, à l'initiative de Jean-Noël Jeanneney et après moult péripéties. Le fonds ne contenait pas d'autres papiers. Le don à la FNSP était assorti d'une clause prévoyant l'édition des lettres avant la consultation en raison de leur intérêt et de leur fragilité : «Dans un premier temps, l'exclusivité de l'exploitation de ce fonds sera réservée à un chercheur chargé d'en assumer la publication totale ou partielle, à la demande et sous la responsabilité de la Fondation nationale des Sciences politiques ${ }^{10}$ ».

Malheureusement, la correspondance est seulement active. Malgré d'importantes recherches, il a été impossible de retrouver les lettres de Lebaudy. Mais ce que Nalèche écrit n'appelle que peu de réponse de la part de son correspondant.

La présentation matérielle est toujours la même ${ }^{11}$ : les feuilles, de petite taille, sont brunes, de même format $(22,5 \times 14 \mathrm{~cm})$, de qualité médiocre et donc assez fragiles. Le papier, acide, a vieilli. Le nombre de feuillets par lettre varie d'un (rarement) à quatre (régulièrement). Chaque feuillet est numéroté. Nalèche écrit recto-verso. Il ne réécrit pas en travers de la lettre, contrairement à une habitude courante à l'époque. Son écriture est 
régulière, habituellement lisible et généralement sans abréviations. En revanche, l'encre, violette, bleue ou brune, est parfois un peu passée. Il écrit à la plume, puis au stylo ${ }^{12}$ :

$\mathrm{Si}$, depuis quelques jours, tu trouves mon écriture changée, ne crois pas que ce soit l'effet d'une attaque et que mon gâtisme ait augmenté. J'en suis toujours à peu près au même point mais j'écris sur mon genou avec un stylo, instrument auquel je ne suis pas fait et dont la plume s'obstine à contrecarrer mes habitudes [19-20 janvier 1919].

Le texte est structuré, présenté avec des alinéas qui respectent l'évolution de la pensée, et justifié. Parfois, mais rarement, Nalèche joint une pièce qui appuie son propos. La correspondance est transmise par la poste. Les enveloppes ne sont pas conservées dans le fonds, à la différence de beaucoup de lettres de la période, qui permettent de reconstituer le parcours géographique du destinataire. La localisation de Lebaudy ne peut donc être identifiée ainsi, mais par des éléments extérieurs, comme son dossier au Service historique de la Défense ${ }^{13}$. Les lettres étaient dépliées et bien classées, par ordre chronologique, dans des enveloppes par mois. Elles n'étaient pas annotées. Le classement, dont l'auteur est inconnu, a été respecté. L'ensemble est maintenant conservé dans sept boîtes d'archives et des pochettes en papier neutre par les Archives d'histoire contemporaine du Centre d'histoire de Sciences Po.

La correspondance couvre la période 1914-1929, avec une seule lettre de septembre 1914 depuis Bordeaux, où le gouvernement s'était retiré et que Nalèche avait suivi. La guerre est donc clairement le motif d'entrée en écriture de Nalèche. Les lettres continuent après la guerre jusqu'au $1^{\text {er }}$ août 1929, date de la mort brutale de Lebaudy, mais elles sont moins fréquentes après guerre car le téléphone était plus présent et les deux hommes se voyaient aussi plus souvent. Le mode de communication s'est transformé avec l'évolution technologique et le retour à la vie normale. Le choix retenu pour l'édition est celui des lettres de la Première Guerre mondiale, de 1914 jusqu'au traité de Versailles inclus, ce qui a le mérite de la cohérence intellectuelle et représente une unité chronologique ${ }^{14}$.

L'ensemble est cohérent et intéressant car il est représentatif d'un lien épistolaire au quotidien sur plusieurs années. Mille vingt lettres ont été recensées et éditées pour les années 1914-1919. Les années les plus riches sont 1917 et 1918, avec presque une lettre par jour. Cela correspond aussi à des périodes importantes de la guerre, mais plus à la période où Lebaudy est engagé, jusqu'en novembre 1916. Neuf feuillets épars n'ont pu être datés et rattachés au reste du corpus.

\begin{tabular}{|c|c|c|c|c|c|c|c|c|c|c|c|c|c|}
\hline & janvier & février & mars & avril & mai & juin & juillet & août & sept. & octobre & nov. & décembre & TOTAL \\
\hline 1914 & & & & & & & & & 1 & & & & 1 \\
\hline 1915 & & & & & & & 1 & 1 & 1 & 9 & 10 & 7 & 29 \\
\hline 1916 & 3 & 6 & 27 & 13 & 29 & 28 & 14 & 31 & 31 & 18 & 12 & 28 & 240 \\
\hline 1917 & 27 & 30 & 28 & 29 & 31 & 28 & 29 & 28 & 26 & 28 & 26 & 27 & 337 \\
\hline 1918 & 27 & 25 & 23 & 26 & 26 & 28 & 27 & 21 & 27 & 30 & 24 & 26 & 310 \\
\hline 1919 & 17 & 25 & 20 & 20 & 21 & & & & & & & & 103 \\
\hline & & & & & & & & & & & & & 1020 \\
\hline
\end{tabular}




\section{4-1919.}

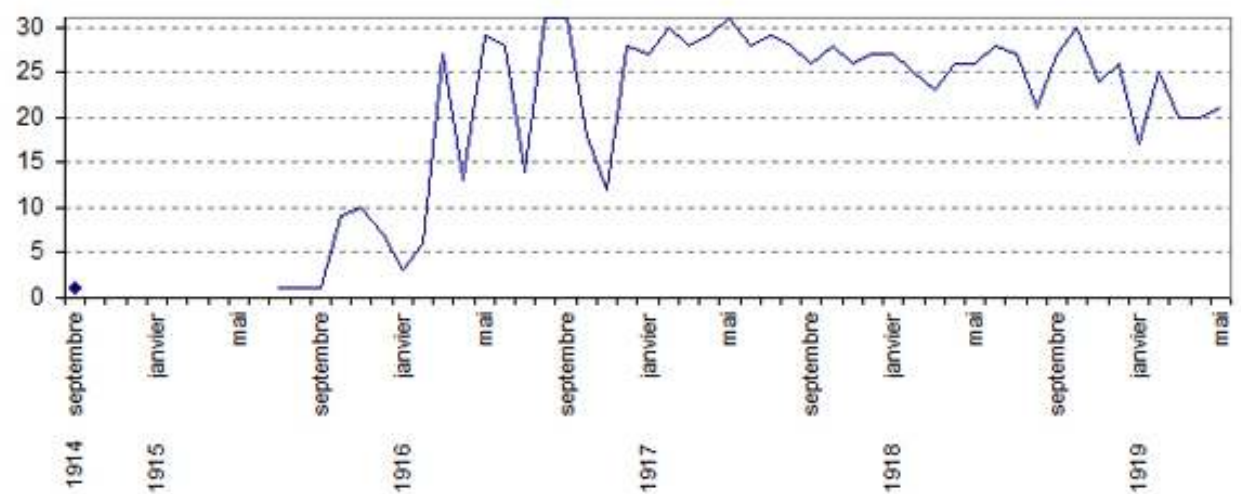

Graphique 1 : La répartition des lettres de Nalèche à Lebaudy par année et par mois, 1914-1919.

La courbe montre bien la différence entre les périodes où Lebaudy est au front et absent de Paris et où Nalèche lui écrit ou en permission et Nalèche ne lui écrit pas et la période postérieure au $1^{\mathrm{er}}$ janvier 1917 où Lebaudy est rentré à Paris et où Nalèche lui écrit presque quotidiennement.

Dans le foisonnement des correspondances éditées et publiées sur la guerre, quelle est l'originalité de celle-ci ? Elle a une double spécificité : son statut et ses apports.

\section{Le statut de ce corpus : de la correspondance à la chronique}

\section{Le pensum : Nulla dies sine linea ${ }^{15}$}

Pendant la guerre, Lebaudy et Nalèche s'inscrivent dans des espaces différents. À partir de 1917, tous deux sont à Paris. Dans la première lettre du corpus, écrite de Bordeaux le 27 septembre 1914, Nalèche se situe, lui à l'arrière, par rapport à Lebaudy qui est au front :

Allons, au revoir, mon vieux Pierre. En pensant à toi je suis presque honteux que mon devoir soit si peu glorieux, mais je tâche de le faire de mon mieux. J'aimerais cent fois mieux être auprès de toi, j'y suis souvent par la pensée, et bien fraternellement je t'embrasse [27 septembre 1914].

Nalèche prend de son temps, une demi-heure environ, pour écrire quotidiennement à Lebaudy. Il conçoit ses lettres comme une obligation vis-à-vis de Lebaudy et de lui-même. Le terme le plus souvent employé pour parler de cette correspondance est celui de "pensum ». Quatre-vingt-trois occurrences du mot apparaissent dans le corpus. Originellement, le pensum est une : " punition à faire, tâche à faire, et au sens propre, le poids de laine que les servantes devaient filer en un jour $»^{16}$. Ici, c'est donc le poids de récit quotidien que Nalèche doit fournir à Lebaudy ! «Il est très tard, mon vieux Pierre, et je ne sais si je pourrai arriver au bout de mon pensum comme tu dis » [4 mai 1916].

Pourquoi Lebaudy a-t-il confié cette mission d'information à Nalèche? Peut-être parce qu'il est un ami proche resté à Paris, qu'il a confiance en lui et l'estime digne de cette 
mission. Probablement aussi parce que Nalèche écrit à l'un des financeurs de son journal, envers qui il est redevable. Nalèche écrit pour remplir un rôle durant cette guerre où lui n'est pas exposé physiquement. Ce serait celui de soutien moral et d'information, celui que peut remplir un homme resté à Paris, proche du pouvoir, intelligent et écrivant bien. Il a gardé trace d'un fragment de vie particulier même si on ne trouve pas d'indication d'utilisation ultérieure par Nalèche lui-même. Il n'a pas fait ce que beaucoup de combattants - il n'en était certes pas un - ont accompli après la guerre : rédiger et publier les notes ou carnets écrits pendant le conflit. Il y a tout de même eu une volonté de conservation puisque les lettres sont revenues dans sa famille, même si nous ne savons pas dans quelles circonstances. Nalèche se rend compte avec lucidité de l'importance de cette guerre pour l'avenir, plutôt que par rapport à lui-même. Cette correspondance n'a pas été rédigée directement pour témoigner, mais pour comprendre et garder trace: «Peut-être que toutes ces histoires t'embêtent, mon pauvre vieux, mais, malgré tout, elles ont leur petit intérêt pour, un jour ou l'autre, arriver à comprendre comment tel ou tel mouvement est né, où tel ou tel fait prend racine » [9 avril 1916].

Cette correspondance est à la frontière de plusieurs genres d'écriture. Plusieurs critères aident à percevoir le statut de ce corpus.

\section{Une correspondance privée?}

21 Le statut de ces écrits est, de prime abord, celui d'une " écriture ordinaire ${ }^{17}$ ", de la correspondance privée entre deux amis, pour un usage personnel et individuel. Notre corpus constitue bien une correspondance au sens défini par Bruno Delmas ${ }^{18}$. La forme est celle d'une lettre manuscrite. Parmi les quatre usages principaux, deux se retrouvent dans notre ensemble de lettres: privé et para-professionnel. L'action est plus souple puisque les lettres de Nalèche remplissent différentes fonctions définies par Bruno Delmas : « informer » bien sûr, et, la plupart du temps, « relater » aussi un événement à la demande de Lebaudy, "demander", par exemple lorsqu'il souhaite du sucre pour ses nièces ${ }^{19}$, "répondre " aux épîtres de Lebaudy, et "transmettre " lorsqu'il renvoie à Lebaudy une autre lettre ou une pièce spécifique. En temps de guerre, la transmission se fait par la poste aux Armées. Nalèche fait fréquemment allusion à la poste, à ses retards et à la censure. Notre scripteur cherche à rester en contact avec son destinataire. Son discours se conforme aux règles implicites de son époque. Sa production est un objet d'écriture et un témoignage intéressant qu'il faut cependant croiser avec d'autres sources.

Il s'agit de lettres, par leur forme et leur structure. Il y a presque toujours une date, une formule d'adresse, de politesse et une signature. Il y a un auteur, Étienne de Nalèche, et un destinataire, Pierre Lebaudy, même si nous ne disposons pas des lettres de Lebaudy en réponse à Nalèche, faisant ainsi que, pour nous, le dialogue est rompu. Il y a donc interaction entre deux personnes qui passe par l'écriture. Nalèche est d'ailleurs, en homme de réseaux, un habitué de la pratique de la correspondance, à titre personnel et professionnel, qui trouve une ampleur encore plus grande pendant la guerre. Il s'agit aussi d'une correspondance car ces documents ont un "caractère d'immédiateté et de spontanéitée ${ }^{20}$ » indéniable malgré l'autocensure. Les lettres sont presque toujours datées et quotidiennes. Nalèche ne fait pas de brouillon, il rédige de manière structurée mais au fil de la pensée et de la plume, et envoie immédiatement ses missives à Lebaudy. Cela en 
fait aussi un document d'archives unique et une source historique fondamentale. Il ne s'agit pas ici d'une réécriture a posteriori mais bien d'un document à chaud.

Cette correspondance est nettement privée dans la forme, notamment les formules d'appel, par exemple «Mon vieux Pierre » ou «Dear Peter » et de politesse comme « crois à ma vieille amitié » ou « ton affectionné » ainsi que le ton général. La continuation d'une amitié de longue date entre deux hommes qui se tutoient - comme de bons camarades de collège qu'ils sont probablement - est le fil conducteur de ces échanges. Mais leur contenu est para-professionnel pour un journaliste comme Étienne de Nalèche et il n'est pas exempt d'intérêts économiques, financiers et professionnels communs, par exemple autour du Journal des Débats dont Lebaudy est actionnaire et administrateur et qu'il finance largement. Plusieurs sphères s'interpénètrent.

Très peu d'évocations personnelles et familiales apparaissent au fil des lettres, par choix délibéré. Cela constitue une grande différence avec la plupart des correspondances familiales ou amicales de l'époque. Nalèche parle ainsi de manière rarissime de sa santé, de ses sentiments ou de sa famille. Les anecdotes personnelles relatées ont juste une valeur d'exemples. C'est l'intérêt général, politique, diplomatique et militaire qui prime. L'intime n'est pas non plus dévoilé. Le moral de Nalèche se déduit de son ton, presque imperceptiblement. Cette correspondance n'est nullement introspective, même si la situation morale en temps de guerre peut apparaitre. Il ne s'agit donc pas à proprement parler d'« écrits du for privé $»^{21}$, qui incluent souvent une dimension personnelle ou familiale. Notre corpus constitue plutôt une correspondance privée amicale et paraprofessionnelle d'intérêt général.

Le témoignage de Nalèche est très éloigné de celui des combattants. Ce n'est pas une correspondance de poilu, ni des carnets de guerre ${ }^{22}$. Il s'agit plutôt d'une correspondance sur la guerre vue de l'extérieur, et en temps de guerre. La censure est très présente et l'autocensure très perceptible, notamment quand Lebaudy est au front, jusqu'en novembre 1916. Mais Nalèche peut néanmoins envoyer à Lebaudy des informations qu'il ne peut écrire dans le Journal des Débats. Cela confirme aussi l'existence de relations régulières entre le front et l'arrière.

\section{Un journal ?}

Finalement, cette correspondance n'est-elle pas aussi ou plutôt une forme de journal ou de chronique, au sens du sire de Joinville ${ }^{23}$, pour garder la mémoire d'événements perçus et analysés comme importants pour l'avenir? La parenté avec le journal est nette car Nalèche s'impose une discipline pour écrire quotidiennement son «pensum»: l'étymologie de «journal» comprend bien la pratique régulière, au jour le jour. La périodicité peut varier, mais elle s'impose. Celle des lettres de Nalèche change d'ailleurs : de quotidiennes quand Lebaudy est au front, elles peuvent être rédigées tous les deux ou trois jours après son retour. Nalèche lui-même accorde de l'importance à la régularité et à la précision des dates.

Béatrice Didier insiste sur le caractère discontinu du journal, qui est aussi celui de la correspondance. Il peut être rédigé dans des situations exceptionnelles, qui permettent une expérience. C'est le cas ici de la guerre. Le journal n'est pas seulement un «dialogue avec soi-même ", mais un " genre ouvert à la présence d'autrui ${ }^{24}$ ", ce qui le rapproche de la correspondance. En tant qu'écrits "pour autrui ", notre correspondance ne tient pas du journal intime mais se rapproche de ce que Michel Tournier appelle « Journal extime ${ }^{25}$ 
» ou que Béatrice Didier définit comme un « journal externe » ou des chroniques, avec un critère de quotidienneté. C'est bien le cas de Nalèche qui note et rend compte quotidiennement à Lebaudy des événements et de leur succession: «Il est facile de comprendre que tous ces généraux soignent certains hommes politiques et se servent de leurs influences pour se démolir les uns les autres. Nous connaissons hélas tout cela. Tu dois en retrouver fréquemment les traces dans les notes que je te griffonne au jour le jour. » [3 juillet 1917].

Pierre-Jean Dufief insiste sur la proximité entre journal et correspondance en raison du statut du destinataire : ce n'est pas ici une écriture intime car Nalèche n'est pas en prise directe avec son Moi intime. Ces lettres jouent plutôt un rôle de transmission, d'intermédiaire entre la société et Lebaudy, voire plus. Mais il s'agit vraiment d'une correspondance car Nalèche tient compte de son ami destinataire. Il envoie aussi à Lebaudy des informations confidentielles qu'il ne peut publier de manière ouverte dans le Journal des Débats. Ne pas posséder la correspondance croisée renforce le sentiment que nous avons affaire à un journal externe, surtout à la fin de la période. C'est un fragment de dialogue, une forme de journal à deux voix dont l'autre s'est éteinte. Cela accentue l'impression que certaines lettres n'attendent pas de réponse, d'autant plus qu'elles ont de moins en moins de formule d'adresse, comme si Nalèche écrivait à un correspondant muet ou fantôme. Les raisons de tenir un journal rejoignent les raisons pour lesquelles Nalèche écrit à Lebaudy. En revanche, Nalèche ne possède plus ses lettres, qui sont entre les mains de Lebaudy. Elles lui échappent. Il ne peut pas les relire car il ne semble pas en avoir conservé de brouillons, même si c'est finalement sa famille qui les a récupérées. Dans le cas d'un journal, la possession physique de l'objet demeure. Le corpus s'apparente à des mémoires au sens de ceux du XVIII siècle qui ont peu de conscience du soi privé. Nalèche a conscience de lui-même, mais ce n'est pas ce qu'il a choisi de faire passer dans sa correspondance. Il emploie l'expression « témoin oculaire " à cinq reprises mais ne s'y inclut pas $^{26}$. En revanche, il est une sorte de témoin moral pendant la guerre au sens où l'entend Jay Winter ${ }^{27}$. Par la pratique et le tempérament de Nalèche, c'est encore une pratique du XIXe siècle. Finalement, c'est une écriture ordinaire en temps extraordinaire.

\section{L'évolution chronologique du statut}

À la lumière des critères que nous venons de présenter, nous constatons qu'une évolution chronologique est perceptible dans le corpus et qu'elle implique finalement une différence de statut selon la période. La correspondance n'a pas tout à fait la même forme et le même contenu quand Lebaudy est au front et quand il revient définitivement à Paris fin 1916 et que les deux amis se voient régulièrement. Durant les permissions de Lebaudy, les lettres s'interrompent deux fois quinze jours en avril et juillet 1916. En revanche, au retour de Lebaudy, en congé à partir du 10 novembre 1916, l'échange épistolaire continue alors que les deux amis se voient ou s'appellent au téléphone, mais glisse vers la conservation d'une trace écrite en raison de l'intérêt de la période, pour garder mémoire des événements survenus. Nalèche continue son "pensum» pour «noter la date». Parfois, il n'a pas le temps d'écrire un jour et il regroupe sous deux ou trois dates son récit. Il insiste lui-même sur la différence avec la période où Lebaudy était au front, et sur le fait que Lebaudy à Paris est aussi bien - voire mieux - informé que lui.

Une autre évolution est perceptible dans le rapport entre les deux hommes, plus direct puisqu'ils se voient. Les formules d'appel et de politesse, très présentes dans la première 
période, disparaissent progressivement à partir du retour de Lebaudy à Paris en novembre 1916. Elles deviennent plus courtes, plus sobres et moins nombreuses à partir d'août 1917. Dès octobre 1917, les formules d'appel disparaissent souvent et la formule de politesse se résume à un bref «À toi ». À partir de juillet 1918, la formule d'appel a totalement disparu.

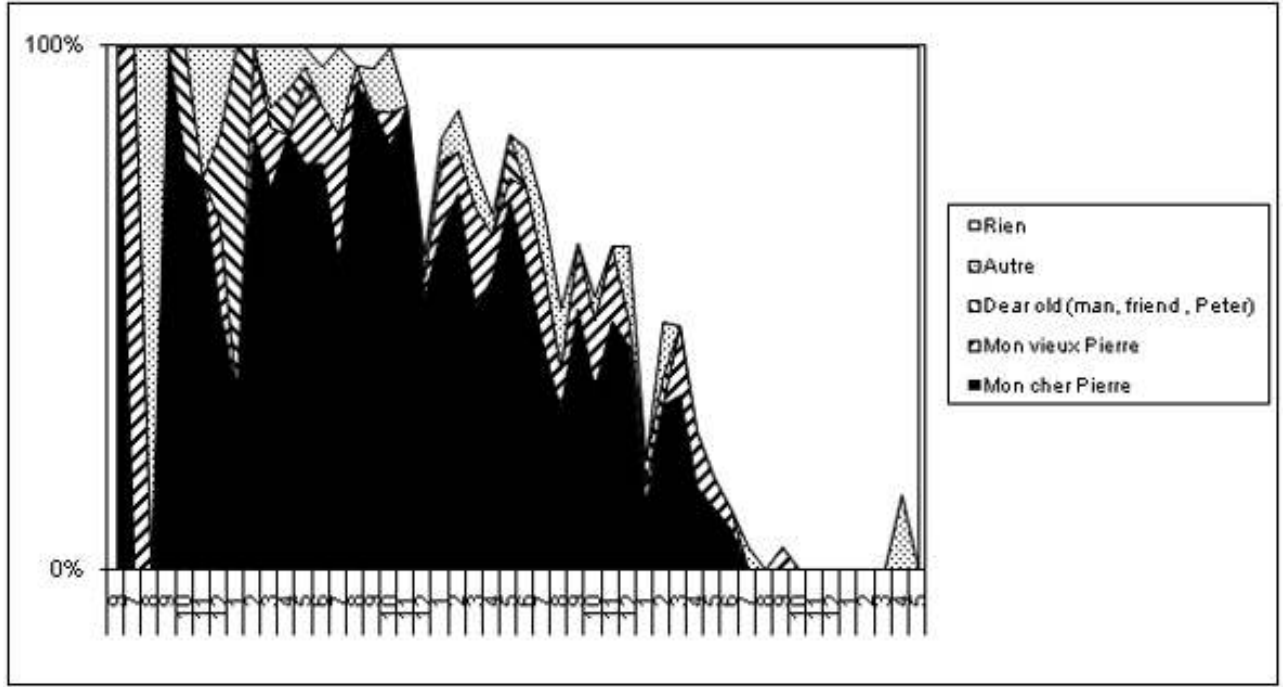

31 Graphique 2: L'évolution des formules d'appel dans les lettres de Nalèche à Lebaudy, 1914-1919.

Ce graphique nous fait percevoir que, à une correspondance réelle, aux attributs bien définis (formules d'appel) au début de la période, quand Nalèche et Lebaudy sont éloignés, a succédé progressivement une forme plus proche de la chronique ou du journal externe, pratiquement sans formule d'appel, parfois au contenu regroupé sous plusieurs dates. D'autre part, la dimension affective disparaît progressivement des lettres au cours de la période. Du début de la guerre, où nous sommes en présence d'une correspondance amicale à un ami éloigné, nous passons à la fin de la période au Journal d'un bourgeois de Paris...

\section{Réflexion épistémologique et fiabilité de la source}

Ce type de source soulève un certain nombre de questions pour une utilisation raisonnée par l'historien, notamment à propos de la fiabilité, de la valeur, des limites et de l'intérêt d'un récit à chaud.

Nalèche se tient informé par de nombreux canaux: correspondants dans les lieux de pouvoir, à Paris, en province et à l'étranger, qu'il connaît bien, contacts personnels avec les hommes politiques, diplomates, professeurs, etc., conversations aux Débats, dans les réunions, les salons et les dîners en ville. Quel crédit peut-on apporter à ces sources respectives, qu'il ne mentionne d'ailleurs pas toujours car il est proche de son correspondant et il procède souvent par allusions ${ }^{28}$ en raison de leur complicité et aussi de la censure. Nalèche aime rapporter des bruits, des rumeurs, des potins, à chaud mais avec un certain esprit critique. Il transmet avec spontanéité à Lebaudy l'information presque telle qu'il l'a reçue et immédiatement, ce qui renforce sa fragilité, car il a peu de recul. Ce sont les mêmes sources que celles qu'il utilise pour son journal, mais il en fait un usage différent. 

part, il possède une grande conscience professionnelle et une haute idée de sa déontologie de journaliste. S'il a écrit une information fausse, une médisance ou une calomnie, il la corrige dès que possible. Quand le croisement avec une autre source est possible, on constate que Nalèche est bien informé. C'est le cas de la scène de Léon Daudet chez Painlevé, que Nalèche relate à Lebaudy ${ }^{29}$ et dont Daudet fait le récit dans ses Souvenirs politiques ${ }^{30}$.

À première vue simple correspondance, mais rédigée dans la durée, ce corpus se transforme de manière imperceptible puis de plus en plus perceptible en un journal destiné à garder mémoire d'un temps hors de l'ordinaire, celui de la guerre, de la rupture dans tous les sens du terme: rupture avec les habitudes quotidiennes, rupture avec le monde du XIX ${ }^{e}$ siècle. Adressée à un destinataire, cette correspondance est aussi mémoire pour son auteur. Le destinataire, Pierre Lebaudy, est un ami, mais il est bien plus, par son statut social, sa richesse et son engagement pendant la guerre. Nalèche lui est redevable à plusieurs titres : Lebaudy s'est engagé et pas lui et il finance son journal. D'autre part, s'il s'agit d'une correspondance privée, le contenu des lettres aborde des sujets publics d'intérêt général dont la portée va bien au-delà de Lebaudy. À ce titre, les thèmes des lettres nous intéressent toujours. Enfin, nous avons vu que cette source était fiable et donc largement utilisable par le lecteur actuel.

\section{Les apports de cette correspondance : la guerre vue de Paris}

Il s'agit donc de mettre à la disposition du public une source originale, différente de la correspondance des poilus, qui concerne la guerre vue de Paris. Les lettres contiennent des informations d'intérêt national d'une richesse extrême, sur des sujets aussi variés que la politique intérieure, la politique étrangère, la diplomatie, la guerre: situation en Irlande, en Roumanie, les questions économiques françaises, les nouveautés militaires (aéronautique, apparition des chars). Nalèche se confond avec son métier de directeur de journal. Il est un observateur flegmatique de la guerre. Il a un réseau très important au service de son journal, il connaît toute l'élite parisienne : diplomates, hommes politiques à qui il sert d'intermédiaire, écrivains, académiciens, directeurs de journaux, hommes d'affaires, autres notables et Tout-Paris, etc.

\section{Une bonne plume}

Dans sa correspondance comme dans son journal, Nalèche a une bonne plume, celle d'un journaliste sérieux, qui a de l'humour, du recul et une grande capacité d'observation et de transmission. Son style est enlevé, expressif et très agréable à lire. Il maitrise l'art de la description comme celui du portrait. La capacité de Nalèche à brosser des scènes touchantes ou poignantes s'illustre par exemple ici, avec sa description des Serbes chassés mourant d'épuisement :

[Un $\mathrm{ami}^{31}$ ] m'a fait une peinture affreuse de l'arrivée des Serbes à Durazzo. Il en a vu venir 10000 et des jeunes recrues se traînant le long des routes, n'ayant sur eux qu'une espèce de manteau des plus grossières étoffes, sans chemises car les Albanais les leur avaient prises en échange de pain et ils s'aidaient dans leur marche de longs bâtons sur lesquels ils s'appuyaient. Beaucoup d'entre eux, épuisés, 
s'asseyaient le long de la route et sans un soupir, sans un cri, s'éteignaient comme des lampes à huile. Il a compté 35 cadavres sur un parcours d'un kilomètre. [12 juin 1916].

À la manière de La Bruyère, Nalèche dresse fréquemment dans ses lettres un portrait vivant et fourmillant de détails des personnages qu'il croise, au physique et au moral. Il a un goût certain pour les anecdotes, notamment croustillantes ou étonnantes, qui émaillent ses lettres ou constituent la chute d'un récit. Elles rendent la correspondance très vivante et servent d'exemple et d'illustration au propos. Elles cherchent également à retenir l'attention du destinataire et à le distraire. Nalèche est lucide et voit l'importance d'un événement, comme les conséquences du bolchevisme. Il touche de près à l'histoire, quand il raconte à Lebaudy comment, à sa salle d'armes, un cercle s'est formé autour du héros Guynemer couvert de médailles ${ }^{32}$. C'est presque alors une image d'Épinal. De même, il évoque la mort de Rostand ${ }^{33}$.

\section{Des sujets « nobles »}

L'étude du contenu des lettres, passées au crible d'un logiciel d'analyse de texte, dénommé Alceste $^{34}$, permet de dégager de grandes catégories lexicales - appelées « classes »- présentes dans les missives ${ }^{35}$. Dans la correspondance de Nalèche, la plupart de ces classes de vocabulaire concernent différents aspects de la vie professionnelle. La correspondance n'aborde que les sujets "nobles" et absolument pas la famille ou les sentiments personnels. Nalèche estime que les thèmes importants et sérieux sont les affaires du pays, la conduite de la politique intérieure et extérieure et la guerre, en priorité. Suivent l'économie et l'aspect social.

41 Avant analyse, le corpus de lettres a été délimité en tranches chronologiques successives (1914-1916, 1917 et 1918-1919) en fonction du volume. Les classes de vocabulaire ont donc été déterminées à l'intérieur de chacun de ces sous-corpus. Des éléments stables durant toute la période se dégagent et d'autres montrent une évolution chronologique. Pour 1914-1916, c'est-à-dire surtout pour les six derniers mois de 1915 et l'année 1916, quatre classes apparaissent. Une première classe regroupe le vocabulaire - lié au type de document analysé - des formules d'appel et de politesse ${ }^{36}$ et des dates ${ }^{37}$. Une deuxième classe concerne la politique intérieure, le gouvernement et le Parlement ${ }^{38}$ et les noms des hommes politiques et des militaires importants ${ }^{39}$. La troisième catégorie rassemble le vocabulaire à dimension internationale et militaire regroupant les noms de pays et de nationalitét ${ }^{40}$, des lieux de bataille ${ }^{41}$ et des termes militaires ${ }^{42}$. La dernière classe a trait à la dimension propre de la guerre et de ses conséquences sanitaires et économiques ${ }^{43}$.

Le vocabulaire repéré par le logiciel n'est pas exactement le même d'une tranche chronologique à l'autre. L'évolution thématique dans le temps est visible à plusieurs traits : en 1917, une classe spécifique vient s'ajouter, consacrée au vocabulaire des affaires journalistico-financières, qui représente $21 \%$ des termes: les protagonistes («Bolo", «Almereyda» et «Lenoir») et le mot «scandale». Parmi les phrases typiques, on retrouve également d'autres morceaux de vie politique traités sur un ton ironique, ainsi une "scène de comédie " lors d'un dîner à Potsdam avec l'Empereur. Les sujets se diversifient encore par la suite puisque six classes sont présentes en 1918-1919, dont une catégorie non négligeable consacrée aux anecdotes $(20 \%)$, peut-être liée à la fin de la guerre. Elle ressemble en partie au vocabulaire des scandales de 1917, mais l'ironie se fait plus légère, les « histoires » ont plus de "charme » (deux mots très présents, comme 
" femme ", « jeune ", " garçon » ou " frère ») : par exemple, « Les dames grecques se sont insurgées contre ce mariage, cependant, la jeune fille est de toute beauté, il faut entendre le grand amateur de femmes, Romanos, décrire cette jeune personne, le type grec dans ce qu'il a de plus parfait ${ }^{44}$.» Une autre classe est tournée vers les enjeux socio-économiques de la guerre, avec des mots comme " argent », « économie », " ouvrier » ou " socialisme » et des phrases comme: «Depuis la guerre, les bénéfices n'existant plus, les ouvriers qui s'étaient habitués à considérer ce supplément comme un dû, s'étaient mis en grève il y a deux ans ${ }^{45} »$.

La catégorie concernant les relations internationales est aussi de plus en plus orientée vers le lexique de la paix. La part relative de la dimension proprement militaire augmente légèrement. Bien entendu, le vocabulaire lui-même évolue, comme les noms de fronts et de batailles, les nationalités et les pays cités en fonction de la situation militaire, et les hommes politiques les plus présents selon les changements politiques ${ }^{46}$. La proportion du vocabulaire ayant trait aux lettres diminue, passant de $13 \%$ (1914-1916) à $9 \%$ (1917) et étant inclus dans une catégorie globale sur le champ lexical de la méthode de travail et ne formant plus une catégorie propre (1918-1919). Cela confirme l'analyse effectuée cidessus sur la diminution des formules d'appel et de politesse et le changement de statut de la correspondance.

Malgré une évolution perceptible des thèmes traités par Nalèche dans sa correspondance, qui reflètent certainement en grande partie ceux de son journal, les sujets abordés révèlent ses centres d'intérêt, d'ordre général et qui concernent majoritairement la politique intérieure, les relations internationales et la guerre, comme le confirme le schéma ci-dessous :

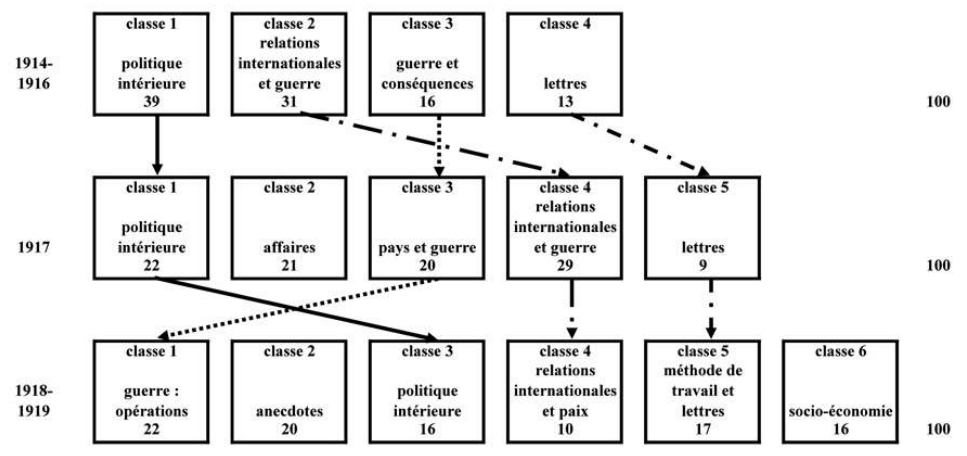

Schéma 1 : L'évolution lexicale dans la correspondance de Nalèche à Lebaudy, 1914-1919. Les données chiffrées sont indiquées en pourcentages par période, la somme faisant 100.

En approfondissant le vocabulaire de chaque classe, il est possible de voir les sujets abordés par Nalèche dans ses lettres. En politique intérieure, il parle de la constitution des cabinets ministériels et des combinaisons possibles, des discours, des interpellations et des débats à la Chambre des députés et au Sénat et du comportement des parlementaires vis-à-vis de qui il est assez critique. Il évoque le fonctionnement des commissions parlementaires et, à partir de 1916, des comités secrets. Les incidents à 
l'Assemblée et les bruits de couloir sont abondamment relatés. Il s'intéresse au comportement, à l'action et aux décisions des ministres et du président du Conseil. Il s'agit donc du fonctionnement de la démocratie et des grands sujets de politique intérieure. Les diverses couleurs politiques sont évoquées. Nalèche est toujours assez hostile aux socialistes, mais il se tient bien au courant, par exemple, de leurs congrès. La rivalité entre les pouvoirs politique et militaire et entre les généraux est visible au fil des lettres. Les personnalités des ministres de la Guerre (Roques, Lyautey, Painlevé et Clemenceau) et des chefs militaires (Joffre, Foch, Gallieni, Pétain, Castelnau, Nivelle, Gouraud, Sarrail, Mangin, Fayolle, Franchet d'Espérey, Micheler, Guillaumat, Mondésir, etc.) se dessinent en filigrane. Le fonctionnement du Grand Quartier général, de la censure et de la Maison de la Presse et des services de propagande apparaît au fur et à mesure des pages.

Les relations internationales sont abordées à travers la diplomatie et la guerre. La situation des ambassadeurs français en poste est fréquemment soulevée, ainsi que leurs changements d'affectation. Les équilibres stratégiques mondiaux sont abordés ainsi que les positions politiques des différents pays et leurs alliances. La position de Nalèche est claire : il est pro-serbe et anti-italien. Il parle beaucoup des pays où le journal possède des correspondants, notamment les pays voisins : Belgique, Italie, Espagne, Grande-Bretagne et Suisse mais aussi les pays d'Europe centrale. Il connaît bien la Grèce. La situation politique et économique au Moyen-Orient figure également parmi ses sujets de préoccupation. Il s'inquiète de l'évolution de la Russie au fil du temps. Les États-Unis sont beaucoup plus évoqués à partir de 1917 et de leur entrée dans la guerre aux côtés des Alliés.

Les opérations militaires figurent en bonne place, même si la vision de Nalèche n'est jamais directe. Il parle des offensives sur les différents fronts (Nord, Est et Orient) et des projets militaires dont il a entendu parler. Les forces en présence et les pertes humaines et matérielles sont abordées, ainsi que la question du moral des troupes. Au moment de la bataille de Verdun, il évoque beaucoup la situation, surtout du point de vue politique, militaire et technique, et très peu du point de vue du quotidien des hommes. La coopération entre les Alliés, dans tous les domaines, est mentionnée. La question des services de santé est bien couverte, entre autres grâce à un informateur de premier plan, Jean de Kergorlay, secrétaire général adjoint de la Croix-Rouge, mais aussi grâce à des familles de blessés, des médecins et des hommes politiques. Nalèche est très critique sur le fonctionnement des services de santé: désorganisation, manque de moyens, responsabilités dans les décès faute de soins, difficultés à retrouver l'ambulance ou l'hôpital où sont transportés les blessés. Il parle également des maladies, de la propagation rapide de la syphilis et de l'apparition de la grippe espagnole. Les œuvres en faveur des blessés sont citées à plusieurs reprises. Les effets de la guerre sur la vie à l'arrière sont signalés : nécessité de laissez-passer, couvre-feu, bombardement de gothas à Paris, mort de civils dans l'effondrement de l'église Saint-Gervais pendant l'office du Vendredi saint 1918. La dimension technique de la guerre n'est pas passée sous silence. On voit apparaître les chars et automobiles blindées, que Nalèche appelle la première fois " crocodiles $^{47}$ » et il dessine même un schéma pour Lebaudy ${ }^{48}$. L'évolution matérielle apparait très clairement, notamment parce que Nalèche sait que Lebaudy, financeur de dirigeables au début du siècle, est friand de ces sujets. Il parle de tous les types d'engins, qu'ils se déplacent par air, mer ou terre (avions, zeppelins, gothas, hydravions, bateaux, sous-marins, trains, automobiles ou chars) et d'armes (canons, mitrailleuses ou obus), 
sous les différents aspects techniques, industriels et militaires mais aborde aussi les débats politiques ou les problèmes administratifs qui leur sont liés. L'évolution de leur utilisation et de leur supériorité au cours de la guerre est appréhendée. Les occurrences de ce type de termes sont extrêmement fréquentes et nombreuses. La correspondance est donc très riche pour ce thème. À partir de 1918, c'est la paix qui est évoquée : laquelle, dans quel délai et à quelles conditions. La venue des délégués à la conférence de la Paix donne lieu à de nombreux dîners auxquels Nalèche est convié, ainsi qu'à des rencontres et des réunions. Les étapes de la conférence à Versailles sont suivies au jour le jour.

Les affaires politico-financières sont régulièrement abordées à partir de 1917. Elles concernent souvent des journaux et ont donc une dimension professionnelle pour le directeur des Débats. L'affaire Caillaux et le scandale du Bonnet rouge sont au cœur de nombreuses lettres. La dimension économico-sociale apparaitt avec le sujet des grèves, en France et en Angleterre, le coût des matières premières pour la nourriture et l'industrie : pain, beurre ou sucre aussi bien que le charbon ou le fer destinés aux chemins de fer, à l'armement et aux usines en général. Le vocabulaire consacré aux anecdotes, qui constitue une classe à partir de 1918, est lié à la personnalité mondaine de Nalèche et aussi une légèreté plus grande dans la perspective de la paix. Les dîners sont relatés avec de nombreux détails. Les scènes sont racontées comme chute à la fin des lettres. Quelques récits croustillants et histoires de familles viennent pimenter le sérieux des relations habituelles, et les bruits et rumeurs, égayer le quotidien de Pierre Lebaudy.

L'intérêt des mille vingt lettres adressées par le directeur des Débats à son ami Lebaudy tient à la fois au statut original et évolutif de la correspondance, qui passe de missives privées et para-professionnelles à un journal gardant la trace lucide de l'importance de la Première guerre mondiale pour l'avenir, mais aussi au contenu évoquant tous les sujets nobles, d'importance première, vus de Paris par de nombreux informateurs et enfin à l'art d'écrire et de décrire de Nalèche, directeur de journal et "gentilhomme » à la frontière de deux siècles.

\section{Annexe : une lettre en exemple : le 16 avril 1917}

La lettre du 16 avril 1917 est assez typique des lettres du corpus. Elle est composée d'une date « 16 avril 1917 », d'une formule d'adresse « Mon cher Pierre », de huit paragraphes, d'une formule d'adieu « Ton vieil », d'une signature « Et », et du post-scriptum.

Sa structure en huit paragraphes se développe ainsi :

- un paragraphe sur les conditions d'écriture du pensum,

- un paragraphe sur la transmission par un informateur sur le conseil des ministres et l'offensive,

- trois paragraphes sur la situation militaire à Saint-Quentin, Soissons-Reims et l'est de Reims, soit un paragraphe par secteur,

- un paragraphe sur la venue de Fernand de Brinon aux Débats pour parler d'une organisation en faveur des civils des régions envahies dans une situation catastrophique,

- un paragraphe sur la démarche entreprise sans succès à ce sujet auprès de Jean Dupuy et du Syndicat de la presse parisienne,

- un paragraphe de conclusion contenant l'avis de Nalèche sur cette détresse.

Un post-scriptum, après la formule de politesse et la signature, fait part d'un bruit (faux) sur la mort de Guynemer.

La lettre est donc très structurée. Elle aborde quatre sujets: le pensum, la situation militaire, la situation des civils et la mort de Guynemer. Trois sur quatre sont des sujets 
«nobles », l'un est lié à la relation entre les deux correspondants. Il y a une opinion de Nalèche et une rumeur fausse. Deux sources sont citées : un informateur et Brinon. Celle de la rumeur n'est pas mentionnée.

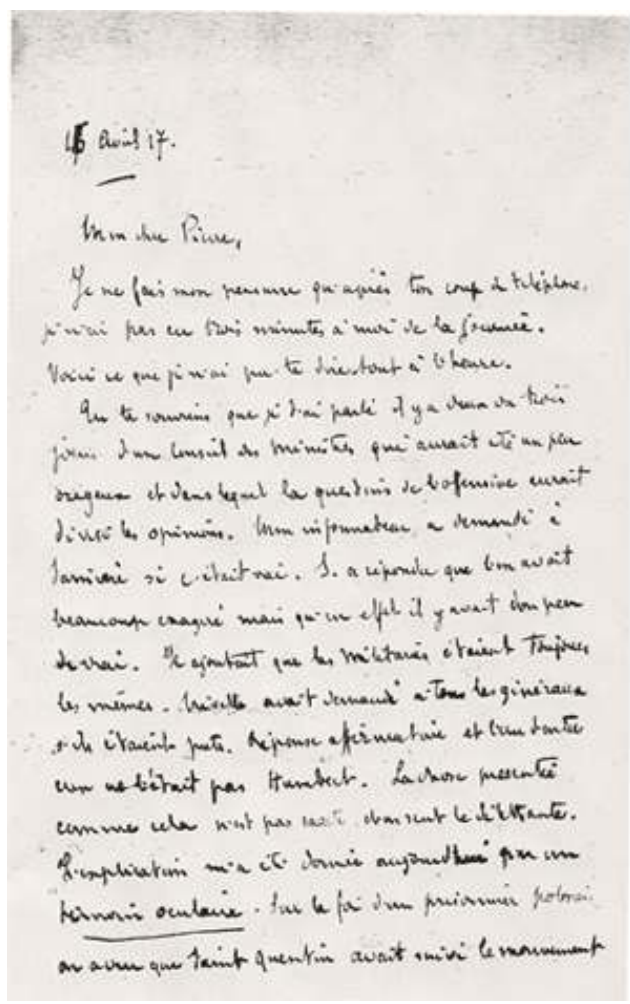

Archives d'histoire contemporaine, Centre d'histoire de Sciences Po (CHSP), fonds Étienne de Nalèche, 16 avril 1917.

Mon cher Pierre,

Je ne fais mon pensum qu'après ton coup de téléphone, je n'ai pas eu trois minutes à moi de la journée. Voici ce que je n'ai pu te dire tout à l'heure.

$\mathrm{Tu}$ te souviens que je t'ai parlé il y a deux ou trois jours d'un Conseil des ministres qui aurait été un peu orageux et dans lequel la question de l'offensive aurait divisé les opinions. Mon informateur a demandé à Saincère si c'était vrai. Saincère a répondu que l'on avait beaucoup exagéré mais qu'en effet il y avait un peu de vrai. Il ajoutait que les militaires étaient toujours les mêmes. Nivelle avait demandé à tous les généraux s'ils étaient prêts. Réponse affirmative, et l'un d'entre eux ne l'était pas, Humbert. La chose présentée comme cela n'est pas exacte et on sent le dilettante. L'explication m'a été donnée aujourd'hui par un témoin oculaire. Sur la foi d'un prisonnier polonais, on a cru que Saint-Quentin avait suivi le mouvement 


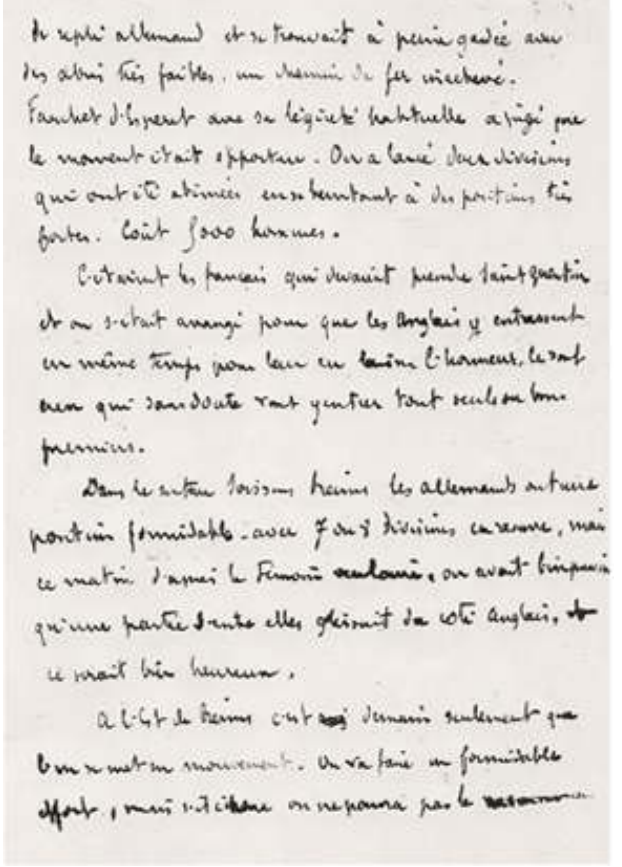

Archives d'histoire contemporaine, CHSP, fonds Étienne de Nalèche, 16 avril 1917.

60 de repli allemand et se trouvait à peine gardée avec des abris très faibles, un chemin de fer inachevé. Franchet d'Espérey, avec sa légèreté habituelle, a jugé que le moment était opportun. On a lancé deux divisions qui ont été abîmées en se heurtant à des positions très fortes. Coût cinq mille hommes.

61 C'étaient les Français qui devaient prendre Saint-Quentin et on s'était arrangé pour que les Anglais y entrassent en même temps pour leur en laisser l'honneur. Ce sont eux qui sans doute vont y entrer seuls en bons premiers.

Dans le secteur Soissons Reims, les Allemands ont une position formidable avec sept ou huit divisions en réserve. Mais ce matin, d'après le témoin oculaire, on avait l'impression qu'une partie d'entre elles glissait du côté anglais, et ce serait bien heureux.

63 À l'est de Reims, c'est demain seulement que l'on se met en mouvement. On va faire un formidable effort, mais s'il échoue on ne pourra pas le recommencer 




Archives d'histoire contemporaine, CHSP, fonds Étienne de Nalèche, 16 avril 1917.

de sitôt.

Le neveu était venu, envoyé par son état-major, pour tâcher d'obtenir une organisation en faveur des pauvres malheureux habitants des régions envahies. Il parait que la misère est épouvantable. Ils n'ont plus rien, ni chaussures, ni habits, ni linge. À Compiègne, dixneuf sont morts de froid. Les militaires s'en fichent comme d'une guigne, et ces pauvres diables commencent à se demander pourquoi on les a délivrés d'un joug qui leur pesait mais sous lequel tout de même ils avaient pu manger si peu que ce soit. Les Allemands les ont expédiés avec un simple paquet de hardes. Rentrés chez eux ils ne trouvent plus rien alors? Le neveu aurait voulu organiser une espèce de centre dans lequel le public aurait envoyé tous les objets matériels et leur état-major se serait chargé de prendre tout cela et de le répartir.

Nous sommes allés trouver Jean Dupuy, mais malheureusement notre Syndicat ne peut rien. 


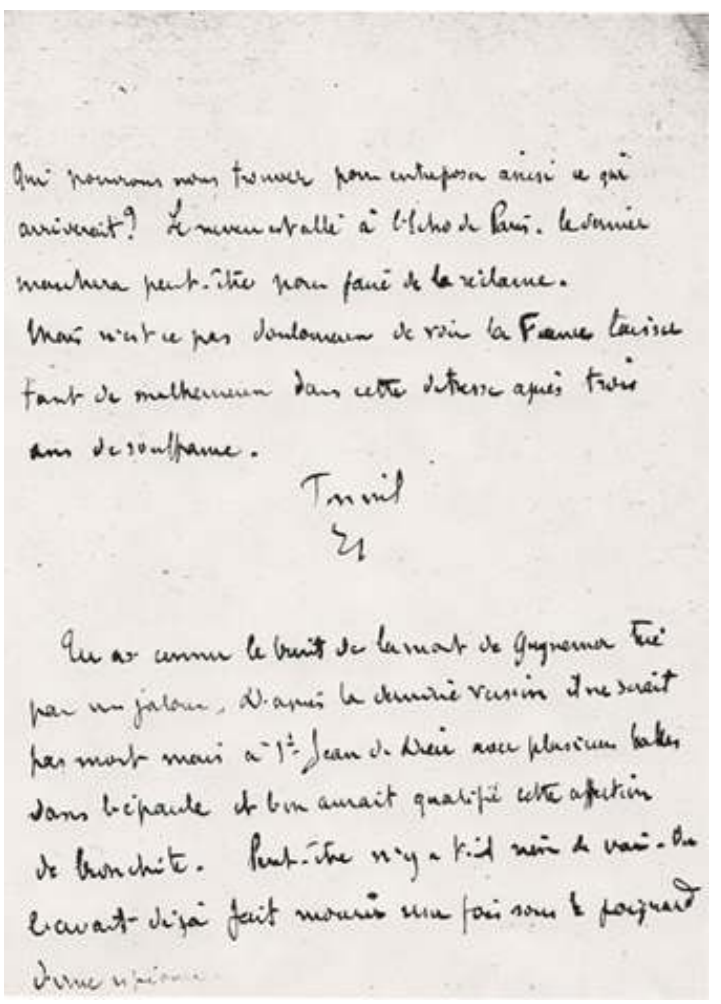

Archives d'histoire contemporaine, CHSP, fonds Étienne de Nalèche, 16 avril 1917.

Qui pourrons-nous trouver pour entreposer ainsi ce qui arriverait? Le neveu est allé à L'Écho de Paris. Ce dernier marchera peut-être pour faire de la réclame.

Mais n'est-ce pas douloureux de voir la France laisser tant de malheureux dans cette détresse après trois ans de souffrance.

Ton vieil

Et

$71 \mathrm{Tu}$ as connu le bruit de la mort de Guynemer tué par un jaloux. D'après la dernière version il ne serait pas mort, mais à Saint-Jean-de-Dieu avec plusieurs balles dans l'épaule et l'on aurait qualifié cette affection de bronchite. Peut-être n'y a-t-il rien de vrai. On l'avait déjà fait mourir une fois sous le poignard d'une espionne.

\section{NOTES}

1. - GAULTIER-VOITURIEZ, Odile. Le "Pensum»: édition critique de la correspondance d'Étienne de Nalèche, directeur du Journal des Débats, à Pierre Lebaudy, industriel sucrier, 1914-1919. Thèse de doctorat, histoire, sous la direction de Jean-Noël Jeanneney, Paris, IEP, janvier 2011. Voir aussi l'édition en ligne : naleche.hypotheses.org.

2. - Mme Pierre Lebaudy a fait créer et installer ce curieux vitrail l'année qui a suivi la mort de son mari, en 1930. Il offre un caractère réinterprété à la mort de Lebaudy, qui, décédé dans un 
accident de voiture dix ans après la fin de la guerre, est représenté en combattant mourant sur le champ de bataille, comme s'il était mort au front. Cela montre le poids de la guerre dans les mentalités longtemps après l'armistice, alors que Lebaudy n'a pas eu de séquelles physiques liées à la guerre. La dimension religieuse reste importante, avec la représentation de la croix du Christ, la présence d'un aumônier militaire et l'installation dans l'église du lieu du décès. Mme Pierre Lebaudy avait certainement des convictions religieuses plus profondes que son mari. Sa participation à la guerre a sûrement beaucoup marqué son épouse. Ce vitrail s'apparente à un exvoto. Voir le site : http://www.culture.gouv.fr/public/mistral/palissy_fr.

3. - « Bien fraternellement je t'embrasse. » [27 septembre 1914].

4. - THOBIE, Jacques. Intérêts et impérialisme français dans l'empire ottoman, 1895-1914. Paris : Publications de la Sorbonne, Imprimerie nationale, université de Paris I, Série Sorbonne, 5, 1977, p. 262-263.

5. - Arch. nat., dossier de Légion d'honneur L 1511090. Voir la base Leonore. Service historique de la Défense (SHD), 5Ye 92857, dossier de Pierre Lebaudy pendant la guerre de 1914-1918 et Croix de guerre.

6. - JEANNENEY, Jean-Noël. François de Wendel en République : l'argent et le pouvoir, 1914-1940, thèse, dir. René Rémond, université Paris-X Nanterre, présentée le 12 mars 1975. Atelier de reproduction des thèses, Lille-III, Honoré Champion, 1976, 3 vol. 1510 p. ; JEANNENEY, Jean-Noël. François de Wendel en République, l'argent et le pouvoir, 1914-1940. Paris : Seuil, L'univers historique, 1976.

7. - MIQUEL, Pierre. « Le Journal des Débats et la Paix de Versailles ». Revue historique, t. CCXXXII, octobre-décembre 1964, p. 379-414 ; MIQUEL, Pierre. La Paix de Versailles et l'opinion publique française. Paris : Flammarion, Nouvelle bibliothèque scientifique, 1972. 8. - JOSEPH, Gilbert. Fernand de Brinon, l'Aristocrate de la collaboration. Paris : Albin Michel, 2002.

9. - Conservé aux Archives d'histoire contemporaine du Centre d'histoire de Sciences Po (CHSP), qui détient des archives d'hommes politiques et de partis politiques.

10. - Article 4 de la convention de don, 14 mai 1997.

11. - Voir annexe : une lettre en exemple : le 16 avril 1917.

12. - Le stylo-plume est inventé par Waterman en 1884. Parker et Sheaffer améliorent les techniques de réservoir et de remplissage comme le remplissage à levier en 1912. Le stylo est utilisé en France à partir de 1907, mais il n'apparaît pas dans le dictionnaire avant 1918. Nalèche, qui écrit beaucoup, adopte donc à la fin de la guerre un nouvel outil.

13. - Service historique de la Défense, $5 Y^{\mathrm{e}}$ 92857, dossier de Pierre Lebaudy pendant la guerre de 1914-1918 et Croix de guerre.

14. - La disparition du mois de juin 1919, probablement demeuré entre les mains de la famille de Pierre Miquel (1930-2007) selon le témoignage de Jean-Noël Jeanneney, imposait aussi cette coupure.

15. - En latin : « Pas un jour sans une ligne ».

16. - ERNOUT, Alfred, et MEILLET, Antoine. Dictionnaire étymologique de la langue latine. Paris : Klincksieck, 1951.

17. - CHARTIER, Roger, et HÉBRARD, Jean. « Conclusion : Entre public et privé : la correspondance, une écriture ordinaire ». Dans CHARTIER, Roger. La correspondance, les usages de la lettre au XIXe siècle. Paris : Fayard, 1991, p. 451-458.

18. - DELMAS, Bruno. « Correspondre : esquisse d'une typologie des formes individuelles et collectives de la communication écrite ». Dans ALBERT, Pierre. Correspondre jadis et naguère. Paris : Éditions du Comité des travaux historiques et scientifiques, 1998, p. 13-29. 19. - Lettre du $1^{\mathrm{er}}$ août 1916. 
20. - BERGAMASCO, Lucia. « Postface ». Dans BOSSIS, Mireille et BERGAMASCO, Lucia. Archive épistolaire et histoire. Paris : Éditions Connaissances et Savoirs, 2007, p. 346.

21. - « Les écrits du for privé, aussi appelés ego-documents, sont des textes non-littéraires produits par des gens ordinaires. Ils vont du simple livre de raison ou livre de famille aux formes plus complexes du diaire, de l'autobiographie, des mémoires historiques et de toutes les sortes de journaux (intime, militaire, de voyage, diplomatique, médical, etc.) », définition donnée par le groupe de recherche GDR 2649 (CNRS) qui associe archivistes et historiens sur les écrits du for privé, malheureusement pour nous jusqu'en 1914 seulement. Voir le site : http://inventaire.ecritsduforprive.fr/. Voir aussi FOISIL, Madeleine. «L'écriture du for privé ». Dans PERROT, Michelle. De la Révolution à la Grande Guerre. Paris : Seuil (Histoire de la vie privée), 1987, p. 331-369.

22. - CAZALS, Rémy. « Éditer les carnets de combattants ». Dans CAUCANAS, Sylvie, CAZALS, Rémy. Traces de 14-18, Actes du colloque, Carcassonne, 24-27 avril 1996.

Carcassonne : Les Audois, 1997. Voir le site : http://www.imprimerie-d3.com/ actesducolloque/editercarnets.html. CAZALS, Rémy, ROUSSEAU, Frédéric. 14-18 : le cri d'une génération : la correspondance et les carnets intimes rédigés au front. Toulouse : Privat, 2001.

23. - Le sire de Joinville (1224-1317), dans les Mémoires de Jean, sire de Joinville, ou Histoire et chronique du roi très chrétien saint Louis, relate sa vie auprès de saint Louis de manière sincère et désintéressée. Quand les sources peuvent être croisées, son témoignage est fiable.

24. - DIDIER, Béatrice. Le Journal intime. Paris : Presses universitaires de France, 1991, p. 24.

25. - TOURNIER, Michel. Journal extime. Paris : Gallimard, 2004.

26. - DULONG, Renaud. Le témoin oculaire : les conditions sociales de l'attestation personnelle. Paris : Éd. de l'EHESS, 1998.

27. - WINTER, Jay. " Le témoin moral et les deux guerres mondiales ». Histoire et sociétés, $\mathrm{n}$ - 8, $4^{\mathrm{e}}$ trim. 2003, p. 99-115.

28. - Dans l'édition critique, trois types de notes ont été rédigés, afin d'éclairer le lecteur : des notes d'identification purement factuelles, pour identifier et situer la personne citée dans le contexte et apporter quelques éléments biographiques directement utiles, ensuite des notes expliquant une allusion peu claire ou un événement (bataille, etc.), et enfin des notes rectifiant une erreur de Nalèche à propos d'un nom, d'une date ou d'une interprétation.

29. - Lettre du 4 novembre 1917.

30. - DAUDET, Léon. Souvenirs politiques réunis par René Wittmann. Paris : Éd. d'histoire et d'art Albatros, 1974, p. 124-125.

31. - Il s'agit d'Alfred Grandidier (1836-1921). Scientifique, géographe, grand voyageur, membre de l'Académie des sciences. Voir le site : France savante/CTHS.

32. - Lettre de [décembre 1915].

33. - Lettre des $1^{\mathrm{er}}-2$ décembre 1918.

34. - Nous tenons à renouveler ici tous nos remerciements à Claire Lemercier pour le traitement d'analyse lexicale auquel elle a soumis le corpus. Alceste est « un logiciel d'analyse de données textuelles issu du CNRS et propriété de la société IMAGE, qui continue à le développer et à le commercialiser depuis 1986. C'est un outil fondamental d'aide à l'analyse automatique des données textuelles. [...] L'objectif est de quantifier un texte pour en extraire les structures signifiantes les plus fortes, afin de dégager l'information essentielle contenue dans les données textuelles». Voir le site : http:// www.image-zafar.com/index_alceste.htm. 
35. - Le logiciel découpe les lettres en unités correspondant à la taille moyenne d'une phrase, puis compare les mots employés dans ces dernières : il regroupe alors les phrases qui emploient les mêmes mots et sépare celles dont le vocabulaire diffère en tout point. Il dégage ainsi des ensembles de phrases qui se ressemblent et, conjointement, des ensembles de mots qui sont souvent employés dans les mêmes phrases. Le logiciel indique les phrases qui, statistiquement, apparaissent les plus typiques de chaque ensemble, de chaque classe de vocabulaire - il s'agit en somme d'une aide à la sélection de citations.

36. - Notamment « je », « te ", «ta », «ton », « mon », « cher », « dear », « vieux », « vieil », « old », « Pierre », « Peter », « revoir », « affectionné » et « lettre ». Une des phrases les plus typiques : « Dear Peter, en te parlant hier de notre ami Denys, j’ai oublié de te mentionner un petit détail qu'il m’a donné sur Verdun. » [29 mai 1916].

37. - Les noms des mois.

38. - Avec des termes comme « ministre/ministère ", " président », « chambre ", « député », « parlementaire », « commission », " comité », les couleurs politiques : « radical », « socialiste/socialisme », les termes techniques associés : « séance », " cabinet ", " discours ", " déclaration », " discussion ». Phrase typique : "Quant à Nivelle, c'est Joffre qui l'a désigné et, d'après la conversation d'Alter ego, il m'apparaît clair comme le jour que la petite affaire montée contre Castelnau est après entente entre Joffre et Roques » [11 décembre 1916].

39. - « Clemenceau », « Briand » ou « Joffre ».

40. - Surtout « allemand », « italien », « bulgare », « anglais », « serbe » et « russe ».

41. - Comme « Verdun ».

42. - En particulier « front », « offensive », « force », « troupe » ou " allié ». Par exemple :

«En réalité, ce que nos alliés veulent, c'est bien marcher mais face au Nord-Ouest, c'est-àdire face à la Serbie, ils ne veulent guère s'en aller face à l'Est » [9 août 1916].

43. - En priorité « soldat », « officier », « compagnie », « camp », « prisonnier », « canon », « propagande », « blesser/blessé », « hôpital », « ouvrier », « usine » et " argent ». Par exemple : «Le service de propagande a eu l'excellente idée d'envoyer un éditeur français s'installer à Zurich pour faire pièce à toutes les œuvres boches qui travaillent dans la ville » [12 mai 1916].

44. - Lettres des 3-4 septembre 1918.

45. - Lettre du 4 octobre 1918.

46. - On passe ainsi par exemple de termes concernant la Roumanie en 1914-1916 à un vocabulaire beaucoup plus important numériquement à propos de la Russie et des ÉtatsUnis, liés à la révolution russe et à l'entrée en guerre des États-Unis.

47. - Lettre du 17 septembre 1916.

48. - Lettre du 20 septembre 1916.

\section{ABSTRACTS}

During the First World War, Étienne de Nalèche, who was the editor in chief of the daily paper, the Journal des Débats remained in Paris. Between 1914 and 1919, every day, he penned a letter to his friend Pierre Lebaudy, member of a wealthy family of industrialists involved in sugar 
production. Despite his advanced years, Lebaudy had managed to be enrolled as an officer. In our article, the context and circumstances of the production of these letters is first presented. We then go on to analyse the corpus of 1,020 letters in all, from one to four pages long, with a structured contents. We examine too how this collection was conserved by the Nalèche family, how several historians have all commented on its remarkable interest and how it ended up in the possession of the Fondation nationale des sciences politiques in Paris (Sciences Po). The originality of the collection lies in the evolution of its status, from a well-written correspondence between two friends to a chronicle of the war, as seen from Paris. The general, political and diplomatic interest of the letters becomes more important than the personal aspects. An example of a letter dated 16 November 1917 is included with the article, in facsimile and with its transcription.

Pendant la Première Guerre mondiale, Étienne de Nalèche, directeur du Journal des Débats, resté à Paris, s'adresse quotidiennement, de 1914 à 1919, à son ami Pierre Lebaudy, industriel sucrier et officier qui s'est engagé malgré son âge. Le contexte et les circonstances de la production sont présentés dans un premier temps. Sont ensuite évoqués le corpus de 1020 lettres d'un à quatre feuillets au contenu structuré, sa conservation dans la famille Nalèche, son utilisation par plusieurs chercheurs qui notent son éminent intérêt et sa transmission à Sciences Po. L'originalité du fonds tient à l'évolution du statut de ce corpus qui, de correspondance entre deux amis, devient une chronique de la guerre vue de Paris: c'est l'intérêt général, politique, diplomatique et militaire qui prime sur les évocations personnelles, le tout écrit d'une plume enlevée. L'exemple de la lettre du 16 novembre 1917 est annexé, en fac-similé et en transcription.

\section{INDEX}

Mots-clés: for privé, correspondance, typologie documentaire, journalisme, Journal des Débats, Première Guerre mondiale, Étienne de Nalèche, Pierre Lebaudy, Paris (France)

Keywords: private writings, correspondence, documentary typology, journalism, First World War

\section{AUTHOR}

\section{ODILE GAULTIER-VOITURIEZ}

responsable du centre de documentation, Centre de recherches politiques de Sciences Po (CEVIPOF) odile.gaultiervoituriez@sciencespo.fr 